\title{
Formal Properties of Metrical Structure
}

\author{
Marc van Oostendorp \\ Werkverband Grammaticamodellen \\ Tilburg University \\ P.O.Box 90153 \\ 5000 LE Tilburg \\ The Netherlands \\ oostendo@kub.nl
}

\begin{abstract}
This paper offers a provisional mathematical typology of metrical representations. First, a family of algebras corresponding to different versions of grid and bracketed grid theory is introduced. It is subsequently shown in what way bracketed grid theory differs from metrical theories using trees. Finally, we show that there are no significant differences between the formalism of bracketed grids (for metrical structure) and the representation used in the work of [Kaye, et al., 1985], [1990] for subsyllabic structure.
\end{abstract}

\section{Introduction}

The most well-known characteristic of Non-linear Phonology is that it shifted its attention from the theory of rules (like in [Chomsky and Halle, 1968]) to the theory of representations. During the last decade phonologists have developed a theory of representations that is sufficiently rich and adequate to describe a wide range of facts from the phonologies of various languages.

It is a fairly recent development that these representations are being studied also from a purely formal point of view. There has been done some work on autosegmental structure (for instance [Coleman and Local, 1991; Bird, 1990; Bird and Klein, 1990]) and also some work on metrical trees (like [Coleman, 1990; Coleman, 1992] in unification phonology and [Wheeler, 1981; Moortgat and Morrill, 1991] in categorial logics). As far as I know, apart from the pioneering work by [Halle and Vergnaud, 1987], hitherto no attention has been paid to the formal aspects of the most popular framework of metrical phonology nowadays, the bracketed grids framework.

Yet a lot of questions have to be answered with regard to bracketed grids. First of all, some authors (for instance [Van der Hulst, 1991]) have expressed the intuition that bracketed grids and tree structures (e.g. the [sw] labeled trees of [Hayes, 1981] and related work) are equivalent. In this paper, I study this intuition in some formal detail and show that it is wrong.

Secondly, one can wonder what the exact relation is between higher-order metrical structure (foot, word) and subsyllabic structure. In this paper I will show that apart from a few empirically unimportant details, bracketed grids are equivalent to the kind of subsyllabic structure that is advocated by [Kaye, et al., 1985], [1990] $]^{1}$.

\section{The definition of a bracketed grid}

Below I give a formal definition of the bracketed grid, as it is introduced by $\mathrm{HV}$ and subsequently elaborated and revised by these authors and others, most notably [Hayes, 1991]. HV have a major part of their book devoted to the formalism themselves, but there are numerous problems with this formalization. I will mention two of them.

First, their formalization is not flexible enough to capture all instances of (bracketed) grid theory as it is actually used in the literature of the last few years. They merely give a sketch of the specific implementation of bracketed grid theory as it is used in the rest of their book. Modern work like [Kager, 1989] or [Hayes, 1991] cannot be described within

\footnotetext{
${ }^{1}$ In this paper, I will use $H V$ as an abbreviation for [Halle and Vergnaud, 1987] and $K L V$ as an abbreviation for [Kaye, et al., 1985], [1990].
} 
this framework.

Secondly, their way of formalizing bracketing grids has very much a 'derivational' flavour. They are more interested in how grids can be built than in what they look like. Although looking at the derivational aspects is an interesting and worthwile enterprise in itself, it makes their formalism less suitable for a comparison with metrical trees.

A grid in the linguistic literature is a set of lines, each line defining a certain subgroup of the stress bearing elements. Thus, in 1 (HV's (85)), the asterisks ('stars') on line 0 represent the syllables of the word formaldehyde, the stars on line 1 secondary stress and line 2 represents the syllable with primary stress:

(1)

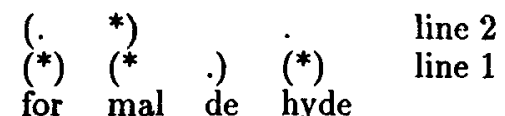

We can formalize the underlying notion of a line as follows:

Definition 1 (Line) $A$ line $L_{i}$ is a pair $\left\langle A_{i}, \prec_{i}>\right.$ where $A_{i}=\left\{a_{i}^{1}, \ldots, a_{i}^{n}\right\}$, where $a_{i}^{1}, \ldots, a_{i}^{n}$ are constants, $n$ a fixed number

$\prec_{i}$ is a total ordering on $A_{i}$ such that the following axioms hold.

a. $\forall \alpha, \beta, \gamma \in L_{i}: \alpha \prec_{i} \beta \wedge \beta \prec_{i} \gamma \Rightarrow \alpha \prec_{i} \gamma$ (transitivity)

b. $\forall \alpha, \beta \in L_{i}: \alpha \prec_{i} \beta \Rightarrow \neg\left(\beta \prec_{i} \alpha\right)$ (asymmetry)

c. $\forall \alpha \in L_{i}: \neg\left(\alpha \prec_{i} \alpha\right)$ (irreflexivity)

We say that $L_{i} \subset L_{j}$ if $A_{i} \subset A_{j}, \alpha \in L_{i}$ if $\alpha \in A_{i}$. Other set theoretic expressions are extended in a likewise fashion.

Yet this formalisation is not complete for bracketed grids. It has to be supplemented by a theory about the brackets that appear on each line, i.e. by a theory of constituency and by a theory of what exactly counts as a star on a given line.

We have exactly one dot on top of each column of stars $^{2}$.Moreover, each constituent on a line has one star in it plus zero, one or more dots. The stars are heads. HV say that these heads govern their complements. This government relation can only be a relation which is defined in terms of precedence. Suppose we make this government relation into the primitive notion instead of the constituent. A metrical line is defined as a line plus a government relation on that line:

Definition 2 (Metrical line) $A$ metrical line $M L_{i}$ is a pair $<L_{i}, R_{i}>$, where

$L_{i}$ is a line

$R_{i}$ is a relation on $L_{i}$ and an element of $\left\{\prec_{i}, \succ_{i}, \rightarrow_{i}\right.$ $\left., \leftarrow i, \sim_{i}\right\}$

\footnotetext{
${ }^{2}$ That is, if we follow the current tradition rather than
}

With the following definitions holding ${ }^{3}$ :

Definition 3 (Precedence Relations) $\alpha \succ_{i} \beta \Leftrightarrow$ $\beta \prec_{i} \alpha$

$\alpha \leftarrow_{i} \beta \Leftrightarrow \alpha \prec_{i} \beta \wedge \neg \exists \gamma:\left[\alpha \prec_{i} \gamma \wedge \gamma \prec_{i} \beta\right]$

$\alpha \rightarrow_{i} \beta \Leftrightarrow \beta \leftarrow_{i} \alpha$

We assume that something like Government Requirement 1 holds, just as it is assumed in HV that every element is in a constituent, modulo extrametricality (which we will ignore here).

Government Requirement 1 (to be revised below) A line $L_{i}$ meets the government requirement iff all dots on $L_{i}$ are governed, i.e. a star is in relation $R_{i}$ to them.

Now a constituent can be defined as the domain that includes a star, plus all the dots that are governed by this star. We have to be a little bit careful here, because we want to make sure that there is only one star in each constituent.

In a structure like the following we do not want to say that the appointed dot is governed by the first star. It is governed by the second star, which is nearer to it:

(2) $* \ldots * \ldots$

In order to ensure this, we adopt an idea from modern GB syntax, viz. Minimality, which informally says that an element is only governed by another element if there is no closer governor. The definition of Phonological Minimality could look as follows:

Definition 4 (phonological government) $\alpha G_{i} \beta$ ( $\alpha$ governs $\beta$ on line i) iff $\alpha$ is a star and $\alpha R_{i} \beta \wedge \neg \exists \gamma, \gamma$ a star : $\left[\gamma R_{i} \beta \wedge \alpha R_{i} \gamma\right]$

We will give the formal definition of a star later on in this chapter. The government requirement is now to be slightly modified.

Government Requirement 2 to be revised below $A$ line $L_{i}$ meets the government requirement iff all dots on $L_{i}$ are governed, i.e. a star in $L_{i}$ is in relation $G_{i}$ to them.

We can now formally define the notion of a constituent ${ }^{4}$

\footnotetext{
${ }^{3}$ Actually, HV also use a fifth kind of constituent in their book, viz. one of the form (.* .). Because there has been a lot of criticism in the literature against this type of government, I will not not discuss it here.

${ }^{4}$ The reviewer of the abstract for EACL notices that under the present definitions it is not possible to express the kind of ambiguity that is current in (parts of) bracketed grid literature, where it is not sharply defined whether a dot is governed by the star to its left or by the star to its right. This is correct. It is my present purpose to define a version of bracketed grids that comes closest to trees because only in this way we can see which are the really essential differences between the two formalisms.
} 
Definition 5 (Constituent) $A$ constituent on a line $L_{i}$ is a set, consisting of exactly one star $S$ in $L_{i}$ plus all elements that are not stars but that are governed by $S$.

We now have a satisfying definition of a metrical line. We can define a grid as a collection of metrical lines, plus an ordering relation on them:

Definition 6 (Grid) $A$ grid $G$ is a pair $\langle\mathcal{L}, \downarrow\rangle$, where

$\mathcal{L}=\left\{L_{1}, \ldots, L_{n}\right\}$, where $L_{1}, \ldots, L_{n}$ are metrical lines $\downarrow$ is a total ordering on $\mathcal{L}$, such that $\forall L_{i}, L_{j} \in \mathcal{L}$ :

$L_{i} \downarrow L_{j} \Leftrightarrow\left[L_{i} \subset L_{j} \wedge \forall \alpha, \beta \in L_{i} \cap L_{j}:\left[\alpha \prec_{i} \beta \Leftrightarrow\right.\right.$ $\left.\left.\alpha \prec_{j} \beta\right]\right]$

where $\subset$ is intended to denote the proper subset relation, so $L_{i} \subset L_{j} \Rightarrow \neg\left(L_{i}=L_{j}\right)$.

It is relatively easy to see that $\downarrow$ by this definition is transitive, asymmetric and irreflexive. We also define the inverse operator $\uparrow$ such that $L_{i} \uparrow L_{j}$ iff $L_{j} \downarrow L_{i}$.

The most interesting part of definition 6 is of course the $\downarrow$ ('above')-relation. Look at the grid in (3) (= HV's (77), p. 262):

(3)

$\begin{array}{llll} & & \cdot & \text { line 3 } \\ (. & & *) & \text { line 2 } \\ (* & . & (*) & \text { line 1 } \\ \text { Ten } & \text { ne } & \text { see } & \end{array}$

Each of the lines in this grid is shorter than the one immediately below it, in that it has fewer elements. This follows from elementary pretheoretical reasoning. Every stressed syllable is a syllable, every syllable with primary stress also has secondary stress. We expressed this in definition 6 by stating that every line is a subset of the lines below it. By this statement we also expressed the idea that the elements represented on the higher line are in fact the same things as those represented on the lower lines, not just features connected of these. The second part of the definition says that the relative ordering of the elements in each line is the same as that on the other lines.

Our present definition of a metrical grid already has some nice properties. For example, the Continuous Column Constraint, which plays a crucial role as an independent stipulation in [Hayes, 1991] can be derived from definition 6 as a theorem:

(4) Continuous Column Constraint (CCC): A grid containing a column with a mark on layer (=our metrical line) $\mathrm{n}+1$ and no mark on layer $\mathbf{n}$ is ill-formed. Phonological rules are blocked when they would create such a configuration.

The CCC excludes grids like (5), where $\mathrm{b}$ is present on the third line, but not on the second.

(5) $a \quad b$

$\begin{array}{llll}a & & c & d \\ a & b & c & d\end{array}$

We can formalize the CCC as a theorem in our system:

Theorem 1 (CCC) $\forall \alpha \forall L_{i} L_{j}:\left(\alpha \in L_{i} \wedge L_{i} \downarrow\right.$ $\left.L_{j}\right) \Rightarrow\left(\alpha \in L_{j}\right)$

Proof of theorem 1 Suppose $\alpha \in L_{i}$, suppose $L_{i} \downarrow L_{j}$. Then (by (13)) $L_{i} \subset L_{j}$. Now the standard definition of $\subset$ implies $\forall \chi: \chi \in L_{i} \rightarrow \chi \in L_{j}$. Instantiation of $\chi$ by $\alpha$, our first assumption and Modus Ponens give $\alpha \in L_{i}$.

We can also easily define the notion of a dot and a star, informally used in the above definitions of government.

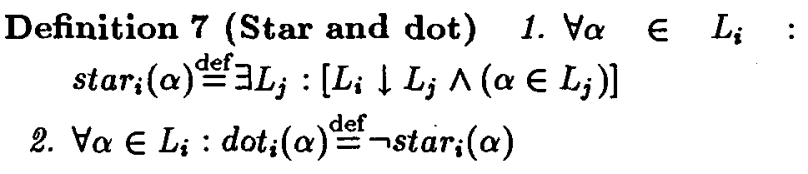

Government Requirement 2 can now be fully formalised and subsequently extended to the grid as a whole.

Government Requirement 3 (for lines) - $f$ nal version $A$ metrical line $L_{i}$ meets the government requirement iff $\forall \alpha \in L_{i}: \operatorname{dot}_{i}(\alpha) \Rightarrow \exists \beta \in L_{i}$ : $\left[\operatorname{star}_{i}(\beta) \wedge \beta G_{i} \alpha\right]$

Government Requirement 4 (for grids) - to be revised below $A$ grid $G$ meets the government requirement iff all lines in $G$ meet the government requirement.

We want to introduce an extra requirement on grids. Nothing in our present definition excludes grids consisting of infinitely many lines. However, in our linguistic analyses we only consider finite constructions. We need to express this. First, we define the notions of a top line and a bottom line. Then we say that a finite grid always has one of each.

Definition 8 (Top line and bottom line) For a certain grid $G, \forall L_{i} \in G$

- $\left(L_{T O P, G}=L_{i}\right) \stackrel{\text { def }}{=} \forall L_{j} \in G:\left[\left(L_{i}=L_{j}\right) \vee\left(L_{i} \downarrow L_{j}\right)\right]$

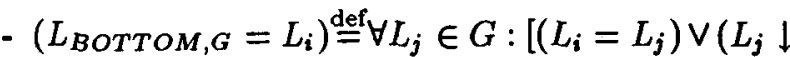
$\left.\left.L_{i}\right)\right]$

Definition 9 (Finite grid) $A$ grid $G$ is called a finite grid if

$\exists L_{i} \in G:\left[L_{i}=L_{T O P, G}\right] \wedge \exists L_{j} \in G:\left[L_{j}=\right.$ $L_{\text {BOTTOM,G] }}$

Note that we have to say something special with regard to the government relation in $L_{T O P, G}$. By definition, this line has only dots in it, so it always looks as something like (6).

(6) .....

There can be no star on this level. A star by definition has to be present at some higher line and there is no higher line above $L_{T O P, G}$. This means that the $L_{T O P, G}$ can never be meeting the government 
requirement and that in turn means that no linguistic grid can ever meet the government requirement. In order to avoid this rather unfortunate situation, we have to slightly revise the definition of meeting the government requirement for linguistic grids.

Government Requirement 5 (for grids) - $f$ nal version $A$ grid $G$ meets the government relation iff all the lines $L_{i} \in G-\left\{L_{T O P, G}\right\}$ meet the government requirement.

Definition 10 (Linguistic grid) $A$ linguistic grid is a finite grid which meets the government relation

A last definition may be needed here. If we look at the grids that are actually used in linguistic theory, it seems that there is always one line in which there is just one element. Furthermore, this line is the top line (the only line that could be above it would be an empty line, but that one doesn't seem to have any linguistic significance).

This observation is phrased in [Hayes, 1991] as follows: if prominence relations are obligatorily defined on all levels, then no matter how many grid levels there are, there will be a topmost level with just one grid mark.

We can formalize this as follows:

Definition 11 (Complete linguistic grids)

A linguistic grid $G$ is called a complete linguistic grid iff $\left|L_{T O P, G}\right|=1$, i.e. $\exists \alpha:\left[\alpha \in L_{T O P, G} \wedge \forall \beta\right.$ : $\left.\left[\beta \in L_{T O P, G} \Rightarrow \beta=\alpha\right]\right]$

We call this type of grid complete because we can easily construct a complete linguistic grid out of every linguistic grid.

If $L_{T O P, G}$ is non-empty, we construct a complete grid by projecting the rightmost (or alternatively the leftmost) element to a new line $L_{i}$ and by adding the government requirement $\succ$ (or $\prec$ ) to $L_{T O P, G}$. Finally we add the relation $L_{i} \downarrow L_{T O P_{i} G}$ to the grid, i.e. we make $L_{i}$ to the new $L_{T O P, G}$.

If the top line of the grid is empty, we remove this line from the grid and proceed as above. Most linguistic grids that are known from the literature, are complete.

Some authors impose even more restrictions on their grids. I believe most of those claims can be expressed in the formal language developed in this section. One example is [Kager, 1989], who claims that all phonological constituents are binary. This Binary Constituency Hypothesis can be formulated by replacing definition 2 :

Definition $12 A$ metrical line $M L_{i}$ is a pair < $L_{i}, R_{i}>$, where

$L_{i}$ is a line

$R_{i}$ is a relation on $L_{i}$ and an element of $\left\{\rightarrow_{i}, \leftarrow_{i}\right\}$

\section{Grids and trees}

In this section, we will try to see in how much bracketed grids and trees are really different formal sys- tems, i.e. to what extent one can say things in one formalism that are impossible to state in the other.

First recall the standard definition of a tree (we cite from [Partee et al., 1990]) ${ }^{5}$ :

Definition 13 (Tree) $A$

(constituent structure) tree is a mathematical configuration $\langle N, Q, D, P, L\rangle$, where

$N$ is a finite set, the set of nodes

$Q$ is a finite set, the set of labels

$D$ is a weak partial order in $N \times N$, the dominance relation

$P$ is a strict partial order in $N \times N$, the precedence relation

$L$ is a function from $N$ into $Q$, the labeling function and such that the following conditions hold:

(a) $\exists \alpha \in N: \forall \beta \in N:[\langle\alpha, \beta\rangle \in D]$ (Single root condition)

(b) $\forall \alpha, \beta \in N:[(<\alpha, \beta>\in P \vee<\beta, \alpha>\in P) \Leftrightarrow(<$ $\alpha, \beta>\notin D \wedge<\beta, \alpha>\notin D)]$ (Exclusivity condition)

(c) $\forall \alpha, \beta, \gamma, \delta:[(<\alpha, \beta>\in P \wedge<\alpha, \gamma>\in D \wedge<$ $\beta, \delta>\in D) \Rightarrow<\gamma, \delta>\in P]$ (Nontangling condition)

It is clear that bracketed grids and trees have structures which cannot be compared immediately. Bracketed grids are pairs consisting of a set of complex objects (the lines) and one total ordering relation defined on those objects (the above relation). Trees on the other hand are sets of simple objects (the nodes) with two relations defined on them (dominance and precedence). These simply appear to be two different algebra's where no isomorphism can be defined.

Yet if we decompose the algebraic structure of the lines, we see that there we have sets of simple objects (the elements of the line) plus two relations defined on them. One of those relations $\left(\prec_{i}\right)$ is a strict partial order, just like $P$. The other relation, $G_{i}$, vaguely reminds us of dominance.

Yet a line clearly is not a tree. Although $\prec_{i}$ has the right properties, it is not so sure that $G_{i}$ does. While this relation clearly is asymmetric (because it is directional), it is not a partial order.

First of all, it is not transitive. (7) is a counterexample.

$$
\text { (7) } \begin{array}{llll}
* & * & - & \leftarrow \\
a & b & c &
\end{array}
$$

Here $a G_{i} b$ and $b G_{i} c$ but not $a G_{i} c$, because of minimality (there is a closer governor, viz. b). $G_{i}$ also

\footnotetext{
${ }^{5}$ For the moment, we will not consider $Q$ and $L$, because these are relatively unimportant for our present aim and goal and there is nothing comparable to the labeling function in our definition of bracketed grids. This is to say that for now we will study unlabeled trees. Notice however that the trees actually used in the phonological literature do use at least a binary set of labels $\{s, w\}$
} 
is irreflexive, of course, because no element is to the left or to the right of itself.

A more interesting relation emerges if we consider the grid as a whole. Because trees are finite structures, we need to consider linguistic grids only. The line $\mathrm{L}_{B O T T O M, G}$ has the property that $\forall \alpha \forall L_{i} \in G$ : [ $\alpha \in L_{i} \Rightarrow \alpha \in L_{\text {Bоттом }, G}$ ]. This follows from the definitions of $L_{B O T T O M, G}$ and of the 'above' relation.

This means that all basic elements of the grid are present on $L_{B O T T O M, G}$ and, as we have seen above, we can equal $P$ to $\prec$ BOTтоM,G. Furthermore, we can build up a 'supergovernment' relation $\mathcal{G}$, which we define as the disjunction of all government relations $G_{i}$ in $G$.

$$
\begin{aligned}
& \text { Definition } 14 \text { (Supergovernment) } \\
& \mathcal{G}_{G} \stackrel{\text { def }}{=} \bigcup_{L_{i} \in G}\left\{\langle\alpha, \beta\rangle \mid \alpha G_{i} \beta \wedge \operatorname{dot}_{i}(\beta)\right\}
\end{aligned}
$$

Again, we exclude the government relation of the two stars in (7).

If we want to compare $\mathcal{G}$ to dominance, we have to make sure it is a partial order. However, $\mathcal{G}$ obviously still is irreflexive. It also is intransitive. Consider the following grid for example.

(8)

$\begin{array}{lllll}\cdot & & * & & \rightarrow \\ a & \dot{b} & c & \dot{d} & \end{array}$

In this grid $a \mathcal{G} c \wedge c \mathcal{G} d$ but $\neg a \mathcal{G} d$. For this reason, we take the transitive and reflexive closure of $\mathcal{G}$, which we call $\mathcal{T} \mathcal{R G}$.

¿From this, we can define the superline of a linguistic grid $^{6}$.

Definition 15 (Superline) The superline $\mathcal{S} \mathcal{L}$ of a linguistic grid $G$ is the tuple

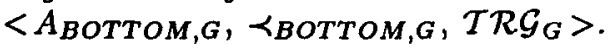

The superline is an entity which we can formally compare to a tree, with $\prec_{\text {Bоттом }}, G=P$, $A_{\text {BotTom, } G}=N, \mathcal{T} \mathcal{R G}=D$. Of most interest are the complete linguistic grids, firstly because these are the ones that seem to have most applications in linguistc theory and secondly because the requirement that they be complete (i.e. their $L_{T O P, G}$ should have exactly one element) mirrors the single root condition on trees. From now on, we will use the abbreviation CLG for 'complete linguistic grid'.

Note that we also restrict our attention to grids which meet the government requirement, i.e. to linguistic grids. We are not so sure that this restriction is equally well supported by metrical theory as the restriction to completeness. However, the restriction

\footnotetext{
${ }^{6}$ The superline itself has no specific status in linguistic theory. I also do not claim it should have one. The superline is a formal object we construct here because it is the substructure of the bracketed grid that comes closest to a tree.
}

to linguistic grids makes sure that all elements in the grid participate in the government relation, because everything ends as a star somewhere and hence has to be governed by another element.

In order to somewhat simplify our proofs below, we introduce one new notational symbol here: $\epsilon$.

Definition 16 (Top Line) $\forall \alpha \in A \forall L_{i} \in G$ : $\left[\alpha \underline{E} L_{i} \Leftrightarrow \alpha \in L_{i} \wedge \neg \exists L_{j}\left[\alpha \in L_{j} \wedge L_{j} \downarrow L_{i}\right]\right]$

This symbol ' $\in$ ' 'top line' denotes the highest line on which a certain element is present. If $\alpha \in L_{i}$, then $L_{i}$ is the highest line at which $\alpha$ can be found. By definition, this means that $\alpha$ is a dot on $L_{i}$.

Of course, for every element in a linguistic grid there is one specific top line.

We now prove:

Theorem 2 For every linguistic grid $G$, if $G$ is complete, then $\mathcal{S} \mathcal{L}_{G}$ satisfies the Single Root Condition.

Proof of theorem 2: Consider a complete linguistic grid $\mathrm{G}$. We have to prove that $\exists \alpha \in A: \forall \beta \in$ $A:\left[\langle\alpha, \beta\rangle \in \mathcal{T} \mathcal{R G}_{G}\right]$ (for shortness, we will refer here and in the following to $A_{\text {BOтT }}$ M,$G$ as $A$ and to $\prec_{B O T T O M, G}$ as $\prec$ where no confusion arises). Consider the (single) element of $L_{T O P, G}$. We call this element $\gamma$ and prove that $\forall \beta \in A:\left[\langle\gamma, \beta\rangle \in \mathcal{T} \mathcal{R G}_{G}\right]$.

(Reductio ad absurdum.) Suppose $\exists \beta \in A:[<$ $\left.\gamma, \beta>\notin \mathcal{T} \mathcal{R G}_{G}\right]$. Because this $\beta$ is in $A, \exists L_{i}:\left[\beta \in L_{i}\right]$. We now take the highest $\beta$ for which this condition is true, i.e.

$\forall \zeta \in A:\left[\langle\gamma, \zeta\rangle \notin \mathcal{T} \mathcal{R} \mathcal{G}_{G} \Rightarrow \exists L_{k}:\left[\zeta \underline{\epsilon} L_{k} \wedge L_{k} \uparrow L_{i}\right]\right]$

$\beta \in L_{i}$ by definition means that there is no $L_{j}$ higher than $L_{i}$ of which $\beta$ is a member. But this in turn means that $\beta$ is a dot on $L_{i}\left(\right.$ or $\left.\operatorname{dot}_{i}(\beta)\right)$.

$L_{i}$ cannot be equal to $L_{T O P, G}$, because in that case we would have $\beta=\gamma$ and since $\mathcal{T} \mathcal{R} \mathcal{G}_{G}$ is reflexive, $\langle\gamma, \beta\rangle \in \mathcal{T} \mathcal{R} \mathcal{G}_{G}$, contrary to our assumption. So $L_{T O P, G} \downarrow L_{i}$

Because $\operatorname{dot}_{i}(\beta)$ and the grid meets the government requirement $\exists \delta:\left[\operatorname{star}_{i} \wedge \delta G_{i} \gamma\right]$. From the definition of supergovernment we then get that $\langle\delta, \beta\rangle \epsilon$ $\mathcal{T} \mathcal{R G}_{G}$

$\delta$ is a star on $L_{i}$. This means that $\exists L_{m}:\left[\delta \in L_{m} \wedge\right.$ $\left.L_{m} \downarrow L_{i}\right]$. We can conclude now that $\langle\gamma, \delta>\epsilon$ $\mathcal{T} \mathcal{R G}_{G}$ holds, because delta is on a higher line than $\beta$ and we assumed $\beta$ was the highest element for which this condition did not hold.

But now we have $\left\langle\delta, \beta>\epsilon T \mathcal{R} \mathcal{G}_{G} \wedge<\gamma, \delta>\epsilon\right.$ $\mathcal{T R \mathcal { G } _ { G }}$ and because $\mathcal{T} \mathcal{R} \mathcal{G}_{G}$ is transitive, $\langle\gamma, \beta>\epsilon$ $\mathcal{T} \mathcal{R}_{G}$. This is a contradiction with our initial assumption.

So superlines have one important characteristic of trees. Yet exclusivity and nontangling still do not hold for superlines of CLGs, even if they meet the government requirement.

A counter example to exclusivity is (9), where $a \prec$ $b \wedge\langle a, b\rangle \in \mathcal{T} \mathcal{R} \mathcal{G}_{G}$. 
(9) $\begin{array}{ll}* & \dot{b}\end{array}$

A counterexample to the nontangling condition is (10), where $a \prec b \wedge\langle a, c\rangle \in \mathcal{T} \mathcal{R} \mathcal{G}_{G} \wedge\langle b, c\rangle \in$ $\mathcal{T} \mathcal{R G}_{G}$ but $c \nprec c$.

(10)

$$
\begin{array}{llll}
* & \cdot & & \leftarrow \\
* & * & \cdot & \leftarrow
\end{array}
$$

The reason why these conditions do not hold is that, on lines as well as on superlines, elements can both govern and precede another element. Exclusivity and nontangling are meant to keep precedence and domination apart.

Sometimes in the literature on trees (e.g. Sampson 1975) we find some weakening of the definition of a tree, in which exclusivity and nontangling are replaced by the Single mother condition.

We first define the mother relation, which is immediate dominance:

Definition 17 (Mother) - to be revised below For all $T, T$ a tree

$\forall \alpha \beta \in T\left[\alpha M \beta \Leftrightarrow<\alpha, \beta>\in D\left[=\mathcal{T} \mathcal{R} \mathcal{G}_{G}\right] \wedge \neg \exists \gamma:[<\right.$ $\left.\left.\alpha, \gamma\rangle \in D\left[=\mathcal{T R} \mathcal{G}_{G}\right] \wedge\langle\gamma, \beta\rangle \in D\left[=\mathcal{T} \mathcal{R} \mathcal{G}_{G}\right]\right]\right]$

Definition 18 (Single mother condition) $\forall \alpha, \beta, \gamma:[(\alpha M \beta \wedge \gamma M \beta) \Leftrightarrow(\alpha=\gamma)]$

Because $\mathcal{T} \mathcal{R G}_{G}$ is the transitive closure of $\mathcal{R} \mathcal{G}_{G}$, we can rephrase defintion 17) as definition 19 for superlines of CLGs:

Definition 19 (Mother) - final version For all superlines $\mathcal{S} \mathcal{L}_{G}$

$\forall \alpha \beta \in \mathcal{S} \mathcal{L}_{G}\left[\alpha M \beta \Leftrightarrow\langle\alpha, \beta\rangle \in \mathcal{R G}_{G}\right]$

We can now prove:

Theorem 3 For every grid $G$, if $G$ is a $C L G$ then $\mathcal{S} \mathcal{L}_{G}$ satisfies the Single Mother Condition.

Proof of theorem 3: (By RAA.) Suppose $\alpha, \beta, \gamma \in$ $\mathcal{S} \mathcal{L}_{G}$ and $\alpha M \beta \wedge \gamma M \beta \wedge \alpha \neq \gamma$. If $\alpha M \beta$, then by definition $19,\langle\alpha, \beta\rangle \in \mathcal{R} \mathcal{G}_{G}$, and if $\gamma M \beta$, similarily $\langle\gamma, \beta\rangle \in \mathcal{R} \mathcal{G}_{G}$. Because $\alpha \neq \gamma$, we have $\alpha \neq \beta$. For if $\alpha=\beta$, we would have $\gamma M \alpha \wedge \alpha M \beta$. But by (19) we then cannot have $\gamma M \beta$. A similar line of reasoning shows that $\beta \neq \gamma$. So $\alpha \neq \gamma \wedge \gamma \neq \beta$. By (17) this means that $\left[\langle\alpha, \beta\rangle \in \mathcal{G}_{G} \wedge\langle\gamma, \beta\rangle \in \mathcal{G}_{G}\right]$, because $\mathcal{R G}_{G}$ is the transitive closure of $\mathcal{G}_{G}$. We have reached the following proposition:

Proposition 1 The mother relation equals $\mathcal{G}$ on superlines: $\forall \alpha \beta \in \mathcal{S} \mathcal{L}_{G}:\left[\alpha M \beta \Rightarrow<\alpha, \beta>\in \mathcal{G}_{G}\right]$

Definition 14 says that if $\langle\alpha, \beta\rangle \in \mathcal{G}_{G}$ there is a line $L_{i}$ such that $\left.\alpha G_{i} \beta \wedge \operatorname{dot}_{i}(\beta)\right\}$. Also, if $\langle\gamma, \beta\rangle \in \mathcal{G}_{G}$ there is a line $L_{j}$ such that $\gamma G_{j} \beta \wedge \operatorname{dot}_{j}(\beta)$ \}. Because $\beta$ can by definition be a dot at exactly one line, $L_{i}=$ $L_{j}$ and $\alpha G_{i} \beta$ and $\gamma G_{i} \beta$. However, from the minimality definition of government (4), it follows that in that case $\alpha=\gamma$. Which is a contradiction.

\section{Dependency Trees}

Let us summarize the results so far. We have seen that from bracketed grids we can extract superlines, on which the government relations of the normal lines are conflated.

These superlines are equivalent to some sort of unlabeled trees, under a very weak definition of the latter notion. Whereas the minimal restrictions of the Single Root Condition and the Single Mother Condition do hold, the same is not necessarily true for the Exclusivity Condition and the Non-Tangling Condition.

It can be shown that in the linguistic literature a form of tree occurs that is exactly isomorphic to bracketed grids. These are the trees that are used in Dependency Phonology.

We did not yet discuss what the properties of these trees are. This is what we will briefly do in the present section.

First let us take a look at the kind of tree we can construct from a given grid. We give the CLG in (11) as an example:

$$
\begin{array}{llllllll} 
& & & & & & & L_{T O P, G} \\
* & j & * & & & * & & \longleftarrow \\
a & b & c & d & e & f &
\end{array}
$$

¿From this grid we can derive a superline $\mathcal{S} \mathcal{L}_{G}$ with $\mathcal{G}_{G}=\{\langle a, b\rangle,\langle c, d\rangle,\langle e, f\rangle,\langle e, a\rangle,\langle$ $e, c>\}$. If we interpret this as a dominance relation and if we draw dominance in the usual way, with the dominating element above the dominated one, we get the following tree:

(12)

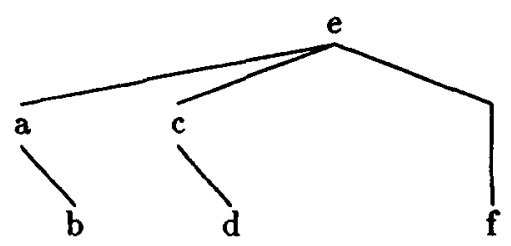

This tree looks rather different than the structures used in the syntactic literature or in metrical work like [Hayes, 1981].

Yet there is one type of structure known in the linguistic (phonological) literature which graphically strongly resembles (12). These are the Dependency Graphs (DGs) of Dependency Phonology ([Durand, 1986] a.o.).

According to [Anderson and Durand, 1986], DGs have the following structure. They consist of a set of primitive objects together with two relations, dependency and precedence. For example within the syllable /s $\mathrm{st}$ / the following relations are holding (notice some of the symbols we introduced above are used here with a slightly different interpretation):

Dependency $s \leftarrow \epsilon \rightarrow t$ (i.e. /s/ depends on $/ \epsilon /$ and $/ t /$ depends on $/ \epsilon /$. 
Precedence $s<\epsilon<\mathrm{t}$ (i.e. /s/ bears a relation of 'immediate strict precedence to $/ \epsilon /$ which, in turn, bears the same relation to $/ \mathrm{t} /$.

Anderson and Durand also introduce the transitive closure of 'immediate strict precedence', 'strict precedence', for which they use the symbol $\ll$ and the transitive closure of dependency, 'subordination', for which they use the double-headed arrow. Moreover, well-formed dependency graphs conform to the following informal characterisation (=Anderson and Durand's (10)):

\section{Definition 20 (Dependency graph) (=Anderson and Durand's (10))}

\section{There is a unique vertex or root}

2. All other vertices are subordinate to the root

\section{All other vertices terminate only one arc}

4. No element can be the head of two different constructions

5. No tangling of arcs or association lines is allowed

20.1 and 20.2 together form a redefinition of the Single Root Condition. Theorem 2 states that superlines of CLGs with the government requirement satisfy this Condition.

Because 'arcs' are used as graphic representations for dependency (which is intransitive), 20.3 seems a formulation of the Single Mother Condition. Theorem 3 states that this condition also holds for superlines of complete linguistic grids.

20.4 needs some further discussion because it is the only requirement that does not seem to hold for our grids. The condition says that something cannot be a head at more than one level of representation, e.g. something cannot be the head of a foot and of a word. However, because of the CCC, in bracketed grid systems the head of a word always is present (as a star - hence as a head) on the foot level.

It is exactly this requirement that is abandoned by all authors of at least Dependency Phonology. [Anderson and Durand, 1986] (p.14) state that one element can be the head of different constructions, as indeed we have already argued in presenting a given syllabic as succesively the head of a syllable, a foot and a tone group.

In order to represent this, a new type of relation is introduced in their system, subjunction. A node $\alpha$ is subjoined to $\beta$ iff $\alpha$ is dependent on $\beta$ but there is no precedence relation between the two.

The word intercede then gets the following representation:
(13)

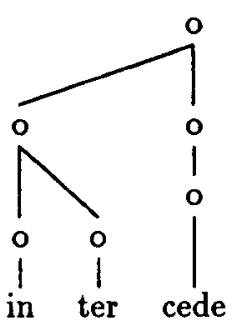

We once again cite [Anderson and Durand, 1986] (p.15):

The node of dependency degree 0 is ungoverned (the group head). On the next level down, at dependency degree 1, we have two nodes governed by the $D D O$ node representing respectively the first foot (inter) and the second foot (cede). The first node is adjoined to the DDO node, the second one is subjoined. Finally, on the bottom level, at DD2, the nodes represent the three syllables of which this word is comprised. These latter nodes are in turn governed by the nodes at DD1 and once again related to them by either adjunction or subjunction.

Lifting the restriction this way seems to be exactly what is needed to fit the superline into the DG formalism.

20.5 holds trivially in the bracketed grid framework as well. It can be interpreted as: if $\alpha$ precedes $\beta$ on a given line in the grid, there is no other line such that $\beta$ precedes $\alpha$ on that line. This is included in the definition of the ' $\downarrow$ ' relation.

The difference between a phonological DG and a bracketed grid is the same as the difference between a superline and a bracketed grid: the DG is not formally divided into separate lines. Interestingly, [Ewen, 1986] analyses English stress shift, one of the main empirical motivations behind the grid formalism, with subjunction. In [Van Oostendorp, 1992b] I argue that using subjunction Ewen's way actually means an introduction of lines into Dependency Phonology.

\section{Government Phonology}

Now let us turn over to a well-known phonological theory that also employs the notion of government as well as 'autosegmental representations'. I refer of course to the syllable theory of KLV and [Charette, 1991].

This theory of the syllable in fact does not have a syllable constituent at all. In stead of such a constituent, KLV postulate a line of $\mathrm{x}$-slots and paralelly, a tier of representation which conforms to the pattern $(O R)^{*}$ - that is an arbitrary number of repetitions of the pattern OR.(KLV [1990]) The term 'tier' suggests an autosegmental rather than a metrical (bracketed grid) approach to syllable structure, but KLV are never explicit on this point.

The fact that $O$ and $R$ appear in a strictly regular pattern can be explained either by invoking the (metrical) Perfect Grid requirement or, alternatively, the 
(autosegmental) OCP. The same applies to the 'labels' $O$ and $R$ : we can define them autosegmentally as the two values of a type 'syllabic constituent' or indirectly as notational conventions for stars and dots on a 'syllable line', i.e. we could have the following representations for KLV's (O R)* line:

$$
\begin{aligned}
& \text { a. tier: } \\
& \text { [type: syll.const; value: } 0, \ldots \text { ] ltype: syll. const; } \\
& \text { value: } R ; \ldots \text { ], etc. } \\
& \text { b. line: } \\
& \quad{ }^{*} \text {, etc. }
\end{aligned}
$$

For (14b), we would have to show that the rhymes or nuclei project to some higher line. We will return to this below.

We still cannot really decide between an autosegmental and a metrical approach. If we look at more than one single line, this situation changes.

At first sight, it then seems very clear that KLV's syllables act as ARs, not as grids. For instance, we can have representations like (15) (from [Charette, 1991]), with a floating Onset constituent:

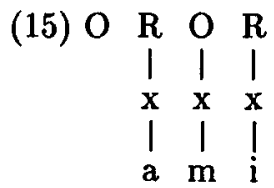

This is a possible autosegmental chart, but not a possible grid (because the Complex Column Constraint is violated by the word initial onset). However, empirical motivation for (15) is hard to find. As far as I know, the structure of (15) is motivated only by the assumption that on the syllabic line we should find $(\mathrm{OR})^{*}$ sequences rather than, say, $(\mathrm{R})(\mathrm{OR})^{*}$.

The same state of poor motivation does not hold, however, to the representation [Charette, 1991] assigns to words with an 'h aspire'?

$$
\text { (16) } \begin{array}{lll}
\mathrm{O} & \mathrm{R} & \\
\mathrm{I} & \mathrm{I} & \\
\mathrm{x} & \mathbf{x} & \mathbf{x} \\
& \text { I } & \text { ! } \\
& \mathbf{a} & \breve{s}
\end{array}
$$

As is well known, the two types of words behave very differently, for example with regard to the definite article. While words with a lexical representation as in (16) behave like words starting with a 'real', overt, onset, words with a representation like (15) behave markedly different:
(17)
a. le tapis - "I' tapis
b. la hache - "l' hache
c. "la amie - l' amie

It seems that, while the empty onset of (15) is invisible for all phonological processes, the same is not true for the empty onset of (16).

\footnotetext{
${ }^{7}$ I disregard the (irrelevant) syllabic status of the final [s] consonant.
}

So there are two different 'empty onsets' in KLV's theory $^{8}$. Notice that the type of empty onset for which there is some empirical evidence is exactly the one where the $(O R)-x$ slot chart does behave like a grid (i.e. where it does not violate the CCC).

So whereas we have here a formal difference between KLV's theory and grid theory, this has no real empirical repercussions.

Another similarity is of course the notion 'government'. For KLV, government only plays a role on the line of $\mathrm{x}$-slots. [Charette, 1991] (p. 27) gives the following summary:

Governing relations must have the following properties:

(i) Constituent government: the head is initial and government is strictly local.

(ii) Interconstituent government: the head is final and government is strictly local.

Government is subject to the following properties:

(i) Only the head of a constituent may govern.

(ii) Only the nuclear head may govern a constituent head.

The most important government relation is constituent government: this is the relation that defines the phonological constituent. Moreover, the 'principles' given by Charette are only introduced into the theory to constrain interconstituent government. By definition, constituent government remains unaffected by these. (As for (i), the definition of the notion constituent implies that it is only the head that governs and (ii) does not apply because we never find two constituent heads within one constituent).

The two conditions on constituent government (that the head be initial and the governee adjacent to it) can be expressed in our formalisation of the grid in a very simple way:

\section{Definition $21 R_{x-s l o t}=\leftarrow$}

According to $\mathrm{KLV}$, $\leftarrow$ is the only possible constituent government relation. Other candidates like $\{\rightarrow, \succ, \prec, \sim\}$ are explicitly rejected, so in fact we have (with some redundancy):

Definition $22 \forall L_{i}:\left[R_{i} \in\{\leftarrow\}\right] \wedge R_{x-\text { slot }}=\leftarrow$

\footnotetext{
${ }^{8}$ [Piggot and Singh, 1985] propose a different distinction, namely one in which the empty onset of ami is represented as (ia) and the one of hache as (ib) (0 is a null segment):

(i) a.

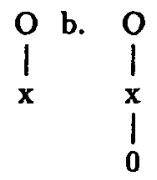

Under this interpretation of Government Phonology, the syllable structure is formally even more similar to grids, if we assume that the linking between segmental material and $\mathbf{x}$-slots has to be outside the grid (treated as autosegmental association) anyway.
} 
This is one of the reasons why KLV do not accept the syllable as a constituent: under their definition of government, this would make the onset into the head of the syllabic constituent.

At least we can see from these definitions that the $x$-slot line in KLV's theory behaves like a normal metrical line.

Yet there is one extra condition defined on this line; this is called interconstituent government. Because of the restrictions in (15), KLV notice that this type of government only concerns the following contexts. (Square brackets denote domains for interconstituent government, normal brackets for constituent government):

(18)

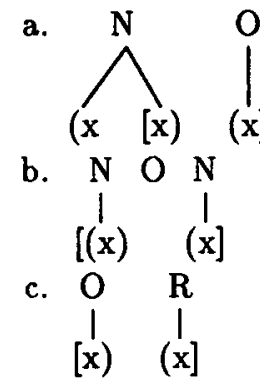

But the fact that there is an extra condition on a line does not alter its being metrical, even if we call this extra condition a government relation ${ }^{9}$.

We now have reached the following representation (20) of the grid variant of the $x$ line in (19) (we use the star-and-dot notation and leave out the association of the autosegmental material to the skeleton):

(19) 0

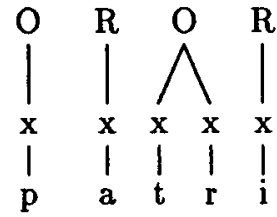

(20)
0
R $\mathrm{O}$
R
$\left({ }^{*}\right) \quad\left(^{*}\right) \quad(* \quad$.
(*)

By definition, stars are present on a higher line. As we have seen above, there is no reason not to consider the $(\mathrm{OR})^{*}$ tier to be this higher line. We then get the following representation:

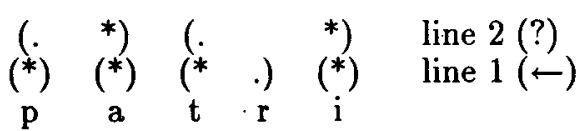

As we noted above, KLV do not accept any constituents on the higher line. One of their reasons was their stipulation that all constituents are left-headed.

There are independent reasons to abandon this restriction. [Charette, 1991] argues for a prosodic analysis of French schwa/ $[\epsilon]$ alternations. In order to do this, she has to build metrical ([s w] labeled) trees representing feet on top of the nuclei. She gives the

\footnotetext{
${ }^{9}$ In [Van Oostendorp, 1992a] I sketch a way of translating 'interconstituent government' to a bracketed grid theory of the syllable.
}

following crucial example [Charette, 1991] (p.180, example (11c)):

(22)

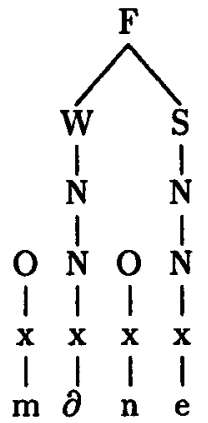

Here we have a clear case of a right-headed phonological constituent, namely the foot.

Furthermore, we see that the nuclei are projected from the $(\mathrm{OR})^{*}$ line to a line where they are the single elements. If we change the top N's in this picture into $\sigma$ 's we have something like a metrical syllable line.

If we incorporate these two innovations into our theory, we can translate th structure in 22 into a perfectly normal grid, in fact into a complete linguistic grid:

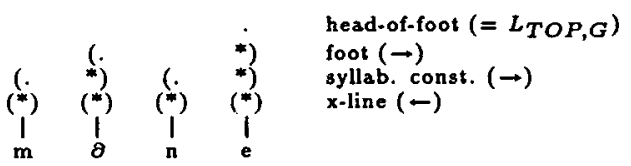

Concludingly, we can say that, although KLV's syllable representations are somewhat different from linguistic grids, two minor adjustments can make them isomorphic:

- in stead of $(\mathrm{OR})^{*}$ we assume $(\mathrm{R})(\mathrm{O} R)^{*}$, i.e. there can be onsetless syllables (KLV themselves note that most of the $(\mathrm{O} R)^{*}$ stipulation can be made to follow from independent stipulations like interconstituent government). This follows a forteriori for the (R) $(\mathrm{O} R)^{*}$ stipulation.

- in stead of 22 we assume $\forall R_{i}:\left[R_{i} \in\{\leftarrow, \rightarrow\right.$ ]] $\wedge R_{x-\text { slot }}=\leftarrow$

The first conjunct of this definition is simply my translation of Kager's ([1989]) Binary Constituency Hypothesis 12 and the second conjunct does the same as the original definition of KLV: it gives the correct choice of government for the subsyllabic line.

As far as I can see, none of these modifications alters the empirical scope of KLV's theory in any important way. I conclude that for all practical purposes, KLV's representation of the syllable equals my definition of a linguistic grid.

\section{Conclusion}

In this paper we have seen that three more or less popular representational systems in modern phonology are notational variants of each other in most 
important ways: these are bracketed grid theory, Dependency Phonology and Government Phonology. The basic ideas underlying each of these frameworks are government/dependency on the one hand and the division of a structure into lines on the other.

The similarity between the frameworks is obscured mainly by the immense differences in notation; but we have shown that the algebraic systems underlying these formalisms is basically the same.

In [Maxwell, 1992] it is shown that the differences between Dependency Graphs and X-bar structures as used in generative syntax are minimal. It remains to be shown whether there are any major formal differences between the bracketed grids that are presented in this paper and the 'X-bar-structures-cum-lines' as they are represented in [Levin, 1985] and [Hermans, 1990].

\section{Acknowledgements}

I thank Chris Sijtsma, Craig Thiersch and Ben Hermans and the anonymous EACL reviewer of the abstract for comments and discussion. I alone am responsible for all errors.

\section{References}

[Anderson and Durand, 1986] John Anderson and Jacques Durand 'Dependency Phonology' In: Durand (1986)

[Bird, 1990] Steven Bird Constraint-Based Phonology Doctoral dissertation, Edinburgh.

[Bird and Klein, 1990] Steven Bird and E. Klein 'Phonological events' Journal of linguistics 29.

[Charette, 1991] Monik Charette Conditions on phonological government Cambridge, Cambridge University Press, 1991.

[Chomsky and Halle, 1968] Noam Chomsky and Morris Halle The Sound Pattern of English. MIT Press, Cambridge (Mass.) 1968.

[Coleman, 1990] John Coleman 'Unification phonology: Another look at sythesis-by-rule.' In: $H$. Karlgren (ed.) Proceedings of COLING 1990. International Committee on Computational linguistics, Vol. 3:79-84.

[Coleman, 1992] John Coleman 'The phonetic interpretation of headed phonological structures containing overlapping constituents' Phonology 9.1 .

[Coleman and Local, 1991] John Coleman and John Local The 'No Crossing Constraint in Autosegmental Phonology' Linguistics and Philosophy 295-338.

[Durand, 1986] Jacques Durand (ed.) Dependency and non-linear phonology Croom Helm, London, 1986.

[Ewen, 1986] Colin Ewen 'Segmental and supersegmental structure' In: Durand (1986)
[Halle and Vergnaud, 1987] Morris Halle and JeanRoger Vergnaud An essay on stress MIT Press, Cambridge (Mass.) 1987.

[Hayes, 1981] Bruce Hayes $A$ metrical theory of stress rules Doctoral dissertation, MIT.

[Hayes, 1991] Bruce Hayes Metrical stress theory: Principles and case studies. Manuscript, UCLA.

[Hermans, 1990] Ben Hermans 'On the fate of stray syllables' In: J. Mascaro and M. Nespor (eds.) Grammar in progress: GLOW essays for Henk van Riemsdijk. Dordrecht, Foris, 1990.

[Van der Hulst, 1991] Harry van der Hulst 'The book of stress' Manuscript, Leiden University, 1991.

[Kager, 1989] René Kager $A$ metrical theory of stress and destressing in English and Dutch Doctoral dissertation, Rijksuniversiteit Utrecht, 1989.

[Kaye, et al., 1985] Jonathan Kaye, Jean Lowenstamm and Jean-Roger Vergnaud 'The internal structure of phonological elements: a theory of charm and government' Phonology yearbook 2:305328.

[Kaye, et al., 1990] Jonathan Kaye, Jean Lowenstamm and Jean-Roger Vergnaud 'Constituent structure and government in phonology' Phonology 7.2

[Levin, 1985] Juliette Levin A metrical theory of syllabicity Doctoral dissertation, MIT. Cambridge (Mass.), 1985.

[Maxwell, 1992] Dan Maxwell 'Equivalences between constituency and dependency.' In: J. van Eijck and W. Meyer Viol (eds.): Computational linguistics in the Netherlands; Papers from the second CLIN-Meeting 1991. Utrecht, OTS, 1992.

[Moortgat and Morrill, 1991] Michael Moortgat and Glynn Morrill 'Heads and Phrases; Type calculus for dependency and constituent structure' To appear in Journal of logic, language and information

[Van Oostendorp, 1992a] Marc van Oostendorp 'The grid of the French syllable' In R. van Hout and R. Bok-Bennema (eds.) Linguistics in the Netherlands 1992. Amsterdam, Benjamins.

[Van Oostendorp, 1992b] Marc van Oostendorp 'The isomorphism between bracketed grid and dependency phonology' Manuscript, Tilburg University.

[Piggot and Singh, 1985] Glynn Piggot and Rajendra Singh 'The phonology of epenthetic segments' Revue Canadienne de Linguistique 30.4:415-453.

[Partee et al., 1990] Barbara Partee, Alice ter Meulen and Robert Wall Mathematical methods in linguistics Dordrecht: Kluwer Academic.

[Wheeler, 1981] Deirdre Wheeler Aspects of a categorial theory of phonology Doctoral dissertation Amherst, 1981. 